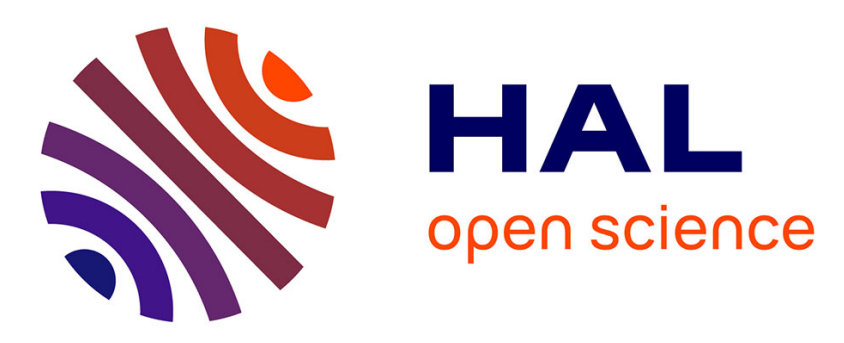

\title{
Carrefours en milieu urbain : quels risques pour les piétons ? Exemple empirique des quartiers centraux de Montréal, Canada
}

Marie-soleil Cloutier, Mathieu Tremblay, Patrick Morency, Philippe Apparicio

\section{- To cite this version:}

Marie-soleil Cloutier, Mathieu Tremblay, Patrick Morency, Philippe Apparicio. Carrefours en milieu urbain : quels risques pour les piétons ? Exemple empirique des quartiers centraux de Montréal, Canada. RTS - Recherche Transports Sécurité, 2014, 2014 (01), pp.3-20. 10.4074/S0761898014001010 . hal-01670624

\section{HAL Id: hal-01670624 \\ https://hal.science/hal-01670624}

Submitted on 21 Dec 2017

HAL is a multi-disciplinary open access archive for the deposit and dissemination of scientific research documents, whether they are published or not. The documents may come from teaching and research institutions in France or abroad, or from public or private research centers.
L'archive ouverte pluridisciplinaire HAL, est destinée au dépôt et à la diffusion de documents scientifiques de niveau recherche, publiés ou non, émanant des établissements d'enseignement et de recherche français ou étrangers, des laboratoires publics ou privés. 


\title{
Carrefours en milieu urbain : quels risques pour les piétons ? Exemple empirique des quartiers centraux de Montréal, Canada
}

\author{
Intersections in urban settings: what is the risk for pedestrians? \\ Empirical example in Montreal central core, Canada
}

\author{
Marie-Soleil Cloutier · Mathieu Tremblay · Patrick Morency · Philippe Apparicio
}

Reçu le 20 décembre 2012 ; accepté le 28 octobre 2013

(C) IFSTTAR et Éditions NecPlus 2014

\begin{abstract}
Résumé Cet article a pour objectif d'évaluer l'impact de variables environnementales que nous appellerons « proximales » (à un carrefour) et « distales » (zone d'influence autour d'un carrefour) sur le nombre d'accidents piétons à ces mêmes carrefours. À partir d'une enquête terrain faite à plus de 500 carrefours des quartiers centraux de Montréal, nous proposons ici de calculer deux modèles de régression : un modèle négatif binomial qui a pour variable dépendante le nombre de piétons blessés dans un rayon de 50 mètres du carrefour ; un modèle logistique ordinal, qui se base sur une variable dépendante avec 3 modalités $(0=0$ accident ; $1=1$ ou 2 accidents ; $2=3$ accidents ou plus). Les résultats suggèrent que la présence de plus de 5 voies, d'un feu de circulation, d'un feu piéton et d'un passage pour piétons (variables proximales), ainsi que le nombre d'emplois par zone d'influence augmentent la probabilité d'avoir plus d'accidents au carrefour ou d'appartenir à une catégorie supérieure d'accidents, à l'exception du passage piéton. À la lumière de ces résultats, il nous apparaît évident que le piéton nécessite plus de considération lors des choix d'aménagement des lieux de traversée, en particulier dans les quartiers centraux de grandes villes comme Montréal.
\end{abstract}

Marie-Soleil Cloutier $(\bowtie)$

INRS-UCS, Urbanisation Culture Société

385 Sherbrooke Est, Montréal, Québec H2X 1E3, Canada

e-mail : marie-soleil.cloutier@ucs.inrs.ca

Mathieu Tremblay $(\bowtie)$

INRS-UCS, Urbanisation Culture Société

385 Sherbrooke Est, Montréal, Québec H2X 1E3, Canada

e-mail : mathieu.tremblay.agence16@ssss.gouv

Patrick Morency $(\square)$

Direction de santé publique de Montréal

1301, rue Sherbrooke Est, Montréal (QUE) H2L 1M3, Canada

e-mail : pmorency@santepub-mtl.qc.ca

Philippe Apparicio $(\triangle)$

INRS-UCS, Urbanisation Culture Société

385 Sherbrooke Est, Montréal, Québec H2X 1E3, Canada

e-mail : Philippe.apparicio@ucs.inrs.ca
Mot clés piétons - aménagement urbain - risque routier . carrefour

\begin{abstract}
This article aims to assess the impact of environmental variables that we call "proximal" (at intersections) and "distal" (in a 1-km-area surrounding intersections) on the number of pedestrian accidents at these intersections. From an data collection at more than 500 intersections in Montréal central neighborhoods, we propose to calculate two regression models: a negative binomial model with the total number of pedestrian injuries (within 50 meters of the intersection) as the dependent variable ; an ordinal logistic model, based on a dependent variable with three categories $(0=0$ accidents $1=1$ or 2 accidents, $2=3$ or more accidents). The results suggest that the presence of more than 5 lanes, the presence of a traffic light, a pedestrian signal and a crosswalk (proximal variables) and the number of jobs around the intersection have a positive effect on the number of accidents at the intersection and on the probability of belonging to a higher category of accidents, with the exception of the crosswalk. In light of these results, it appears obvious that the pedestrian needs more consideration in the urban planning of crossing sites, especially in central districts of large cities like Montréal.
\end{abstract}

Keywords pedestrians · urban planning · road risk . intersection

\section{Introduction}

L'Organisation mondiale de la Santé (OMS) considère les traumatismes dus à des accidents de la circulation comme étant un enjeu mondial de santé puisqu'elle prévoit que cette cause passera du neuvième au troisième rang au niveau des années de vie «corrigées pour les incapacités » d'ici 2030, un indicateur qui tient compte des handicaps et des décès prématurés [1]. De tous ces traumatismes, ceux impliquant une victime piétonne sont particulièrement 
préoccupants puisque les piétons sont généralement blessés plus gravement et hospitalisés plus longtemps [2]. Au Québec comme ailleurs, les piétons qui sont victimes d'une collision se retrouvent principalement dans les grandes villes. À ce titre, en 2009, l'île de Montréal, où nous retrouvons environ $20 \%$ de la population québécoise, représentait près de $50 \%$ de tous les blessés piétons de la province, ce qui en fait un terrain d'étude des plus pertinents lorsqu'il est question de sécurité routière piétonne [3].

Le conflit entre les automobilistes et les piétons n'est pas nouveau, et il semble qu'il ait toujours été générateur de risque. Guillerme [4] nous rappelle que, dès le début du $\mathrm{XX}^{\mathrm{e}}$ siècle, l'avènement de l'automobile dans les villes a donné lieu à une redistribution des espaces publics. La rue a alors été dédiée à ces engins plus puissants que les carrioles à chevaux et les pas des marcheurs. Les piétons sont devenus dès lors une "source d'encombrement " à la fluidité des déplacements. Au cœur de ces conflits entre les piétons et les autres usagers de la route se situe le carrefour, lieu d'intersection des rues, mais surtout lieu de tous les passages où piétons, automobilistes et cyclistes, encore aujourd'hui, doivent respecter certaines règles, qu'elles soient implicites ou explicites, de façon à éviter les collisions. Ce partage « obligé » de la chaussée se fait dans un contexte plus ou moins risqué selon l'environnement en place et les usagers de la route impliqués. En fait, l'aménagement de la voirie aux lieux de traversées peut avoir un certain effet protecteur lorsque les piétons sont séparés à la fois dans l'espace et/ou dans le temps des automobilistes. Cette protection via les aménagements devrait par ailleurs être davantage mise de l'avant aux endroits où le risque est le plus élevé. C'est précisément ce que nous voulons explorer dans le présent article en rapportant, dans un premier temps, les variables environnementales reconnues dans la littérature internationale pour influencer ce risque aux carrefours, et par la suite, en présentant les résultats d'analyses empiriques effectuées à partir d'une enquête terrain exhaustive faite à plus de 500 carrefours des quartiers centraux de Montréal.

\section{Facteurs de risque environnementaux : le cas des piétons dans les travaux en sécurité routière}

Les différents éléments qui influencent le risque ne sont pas toujours faciles à cerner lorsqu'il est question d'accidentologie piétonne. Un récent rapport du Forum International des Transports (FIT) de l'OCDE rappelle d'ailleurs que la marche (et conséquemment les piétons) demeure encore aujourd'hui un mode « oublié » et peu pris en considération dans les processus décisionnels et la sécurité routière urbaine, alors que «la vitalité d'une ville est étroitement liée aux déplacements que les citadins effectuent à pied »[FIT, 5]. Transports Canada insiste : «l'association de personnes non protégées et de véhicules motorisés pouvant rouler vite » constitue le principal facteur de risque chez les usagers vulnérables tels les piétons [6]. Ces évidences nous rappellent que la notion d'exposition au risque est encore plus importante lorsqu'il est question de risque pour les piétons dans l'environnement routier urbain : «En théorie, elle [l'exposition] n'existe que lorsque le piéton et la contrepartie potentielle (un véhicule motorisé ou une bicyclette) se trouvent sur le même espace [c.-à-d. sur la chaussée] » [7]. Ainsi, la première variable à influencer le risque routier est celle du trafic et la littérature internationale nous renseigne depuis déjà plusieurs années sur l'importance des volumes de véhicule dans l'occurrence des collisions $[8,9]$. Par ailleurs, les données de trafic ne couvrent généralement pas tout le réseau routier ni tous les usagers : la collecte de ces données est en soi fastidieuse et leur modélisation difficile, en particulier pour les usagers vulnérables et pour les carrefours avec peu de trafic [10, 11]. Cela a pour conséquence que les études se concentrent habituellement sur un sous-échantillon de sites, comme par exemple les carrefours avec des feux de circulation, modélisant alors un nombre restreint d'accidents. Nous proposons ici de passer en revue les principaux travaux portant sur l'influence de variables environnementales autre que le trafic sur le risque routier piéton afin de contextualiser l'objectif principal du présent article qui est d'évaluer l'impact de certaines de ces variables sur le nombre d'accidents piétons aux carrefours.

Dans le but de mieux comprendre les facteurs de risque environnementaux autres que le trafic, certains travaux de la dernière décennie se sont intéressés aux éléments de l'aménagement des réseaux routiers et des carrefours qui modifient l'exposition des usagers du réseau routier, comme ceux séparant les piétons et les véhicules dans le temps (ex. : feux de circulation à phasage exclusif) ou dans l'espace (ex. : passerelles pour piétons) [12]. La présence d'aménagements dédiés au contrôle des flux automobiles et piétons ou encore la concentration dans un espace restreint de générateurs de déplacement (pôle d'emplois, rues commerciales) créent des opportunités mais aussi des contraintes lors des traversées, ce qui modifie tout autant l'exposition et réduit (ou augmente) le risque d'accident. Ces variables reliées à l'environnement routier agissent à deux échelles : celle « proximale », directement au carrefour et celle « distale », dans une certaine zone d'influence entourant les carrefours. L'originalité des travaux présentés ici tient justement à l'étude simultanée de ces deux sous-groupes de variables sur l'ensemble des accidents répertoriés aux carrefours à l'étude, incluant tous les types de carrefour.

Le premier sous-groupe de variables environnementales rapportées dans les travaux rassemble les différents aménagements présents directement aux carrefours (échelle 
proximale), ce qui inclut tous les outils de régulation des flux et des interactions entre les véhicules ou entre les autres usagers de la route et les véhicules ou encore la signalisation et le marquage. Un des premiers liens explorés dans la littérature passe par l'évaluation de l'impact de différents types de signalisation, bien que les résultats divergent sur la diminution ou non du risque d'accident piéton en présence de ces aménagements. Un article récent de Rifaat et al. [13] a démontré, à partir de modèles de régression logistique, que la probabilité qu'un accident à un carrefour soit mortel pour l'usager vulnérable (piétons et cyclistes), comparé aux accidents sans blessure, décroît de $92 \%$ en présence de feux de signalisation pour les véhicules. Persaud et al. [14] ont quant à eux observé une diminution de $18 \%$ des accidents impliquant un piéton lorsque les feux de circulation ont été remplacés par des panneaux d'arrêt à toutes les approches. Dans le même sens, Pulugurtha et al. [15] ont exploré les différences dans l'accidentologie à 106 carrefours avant et après l'installation d'un décompte pour piéton. Leurs principaux résultats montrent une diminution significative du nombre d'accidents, tous types confondus (véhicules et/ou piétons), mais la diminution pour les accidents impliquant uniquement un piéton et un véhicule n'est pas significative statistiquement. Le marquage au sol de passage piéton est aussi une mesure simple et couramment utilisée par les autorités pour faciliter la traversée. Les rares études sur ce type d'intervention ne peuvent confirmer leur effet protecteur, bien au contraire, puisque certaines d'entre elles associent la présence d'un passage à un nombre plus élevé de piétons blessés, en particulier lorsqu'il n'y a pas de feu de circulation et sur des rues à plus de deux voies [16-18].

Un second lien étudié à l'échelle proximale est celui entre la géométrie et/ou les ajouts "physiques » constituant les carrefours et le risque d'accident piéton. Tout d'abord, la largeur et le nombre de voies à traverser pour le piéton sont des éléments reconnus pour leur impact sur le risque : plus ce nombre est important, plus le temps d'exposition du piéton sur la chaussée est grand et plus il y a de risque de collision [19, 20]. Torbic et al. [21] ont aussi confirmé que la fréquence de collisions entre une voiture et un piéton est plus élevée aux carrefours ayant un plus grand nombre de voies à traverser. Des analyses récentes effectuées à Montréal ont confirmé cette tendance puisque Morency et al. [22] ont démontré que la largeur de la route, la longueur de la traverse piétonne et le nombre de voies de circulation sont associés au nombre moyen de piétons blessés aux carrefours. À l'inverse, la présence de mesures d'apaisement de la circulation aux carrefours ou à l'échelle d'un quartier est de plus en plus reconnue comme étant un moyen efficace de réduire le risque d'accident chez les piétons et les autres usagers de la route [23]. Dans leur classification de différentes mesures d'apaisement, Zein et al. [24] affirment que ces aménagements diminuent de $30 \%$ à $80 \%$ les fréquences de collisions à des sites spécifiques. Retting et al. [12] et Grayling et al. [25] en viennent à la même conclusion, en insistant sur l'efficacité de certaines politiques et mesures particulières comme les zones de rencontre (à basse vitesse), le terre-plein (îlot de refuge central, qu'il serve à une traversée en deux temps ou non) ou le giratoire, les exemples dont il est question dans ces publications étant présents à la fois en Europe (Royaume-Uni, Pays-Bas), au Canada et aux États-Unis. Au Québec, plusieurs initiatives d'apaisement de la circulation sont en place depuis quelques années, mais très peu d'études ont été menées sur leurs impacts en termes d'accidents. Nous pouvons par ailleurs affirmer que les mesures les plus fréquemment choisies par les municipalités sont le ralentisseur (aussi appelé " dos-d'âne ») et la saillie de trottoir [26].

Le second sous-groupe de variables se rattache à une zone d'influence autour du carrefour, c'est pourquoi l'on y fait référence comme étant des variables «distales ». Ces variables sont reconnues pour leur impact indirect sur le risque routier via, encore une fois, l'exposition. La présence d'artères majeures est une de ces variables indirectes qui est fréquemment utilisée dans des modèles de régression tentant d'expliquer l'accidentologie piétonne. Ces artères, parfois utilisées comme proxy du trafic, présentent un lien significatif avec le nombre d'accidents à un carrefour ou dans un territoire donnés. Les travaux qui ont exploré l'effet de la présence de cette variable démontrent que le taux de blessés est plus élevé sur les artères, tout comme il y a une surreprésentation des artères parmi les sites où des piétons ont été blessés [27-32]. Il est à noter ici qu'il n'existe pas une définition unique de ce qu'est une « artère »: chaque entité responsable du réseau routier semble avoir sa propre définition de ce qu'elle représente, bien qu'un certain consensus se dégage sur ce que ce sont des segments du réseau qui ont un trafic élevé (au Québec : un DJMA ${ }^{1}$ $>30000$ véhicules/jour); qui ont entre 4 et 8 voies; dont la vitesse affichée est entre 50 et $70 \mathrm{~km} / \mathrm{h}$; et dont la circulation est contrôlée par des feux optimisés [33].

Pour leur part, les variables de connexité du réseau routier, calculées à partir d'éléments de la forme urbaine comme la présence de « nœuds » (croisements et/ou carrefours) ou la longueur des îlots ou des segments de rue, ont un double effet lorsqu'il est question de risque pour les piétons. Tout d'abord, une plus grande connexité se traduit habituellement par une augmentation du volume de circulation : il est plus facile de naviguer dans des rues dessinées selon un plan en damier que dans celles en cul-de-sac, les trajets sont plus courts et il y a un plus grand

\footnotetext{
${ }^{1}$ Débit journalier moyen annuel.
} 
nombre de trajets directs possibles [34-37]. À l'inverse, le développement d'un réseau de rue en cul-de-sac offre une plus faible connexité et oriente la circulation de transit vers de grands axes de transport à l'extérieur des rues locales [38]. Deuxièmement, une forte connexité facilite la tâche des marcheurs, ce qui en fait augmenter le nombre, surtout lorsque cette connexité se conjugue avec la présence d'une certaine densité populationnelle, de pôle d'emplois ou d'une diversité de destinations intéressantes (rues commerçantes, services de proximité, etc.). Ces deux effets ont pour conséquence une augmentation de l'exposition entre les piétons et les véhicules, augmentant ainsi le risque de conflits, voire d'accidents.

D'autres variables reliées à l'occupation du sol et mesurées par différents indicateurs (taux d'emplois, mixité des fonctions résidentielles et commerciales, densité de population, etc.), ont été utilisées à titre de prédicteurs du nombre d'accidents dans différents modèles de régression, bien qu'elles soient plus souvent utilisées comme prédicteur de la marche utilitaire [39-42]. Par exemple, Wier et al. [43], ont trouvé que le nombre de résidents, le nombre d'emplois et la proportion du sol zoné commercial ou mixte (habitation et commerce) des quartiers sont des variables positivement associées au risque d'accident impliquant un piéton. Pareillement, Moudon et al. [44] indiquent qu'une plus grande densité de population augmente le risque de blessures et de décès chez les piétons.

Bien que les travaux cités précédemment aient tous démontré un lien entre certaines variables proximales ou distales et le risque piéton, très peu d'entre eux se sont intéressés à ces variables de façon simultanée. Nos travaux se positionnent dans cette littérature en ayant un double objectif : de vérifier l'impact relatif de certaines infrastructures routières propres aux carrefours (variables proximales), tout en contrôlant indirectement l'influence des flux (trafic routier et piéton) selon la présence d'éléments du cadre bâti reconnus à la fois pour leur influence sur le risque piéton et sur l'activité piétonne et véhiculaire (variables distales). À partir des travaux recensés, nous avons émis l'hypothèse que la présence d'aménagements pourrait avoir un effet « protecteur » pour les piétons.

\section{Méthodologie}

\section{Données et aire d'étude}

Devant le peu de données et d'exemples empiriques décrivant les différents types d'aménagements reliés à la sécurité des piétons aux carrefours à l'échelle de quartiers ou d'une ville, un effort conjoint de plusieurs partenaires a permis d'effectuer une enquête terrain (été 2009) sur un échantillon représentatif de 508 sites dans 13 quartiers centraux de l'agglomération montréalaise [45]. Les carrefours visités ont été choisis aléatoirement à partir d'une stratification tenant compte de la hiérarchie fonctionnelle des rues, celle-ci provenant du fichier géographique du réseau routier de la région métropolitaine de Montréal [46]. Ce fichier, aussi appelé réseau routier hiérarchique, a été construit à partir de critères touchant la circulation et l'urbanisme et il sert principalement aux planificateurs et gestionnaires du réseau routier montréalais [47]. Les arrondissements et les municipalités de notre territoire d'étude ont tous une responsabilité partagée quant à la voirie et à la signalisation routière : ce partage est fait en fonction de la hiérarchie routière, donnant juridiction à la ville-centre (Montréal) dans le cas des artères et de certaines collectrices; et au ministère des Transports du Québec dans le cas des routes nationales et autoroutes. Tous les autres segments du réseau routier sont sous juridiction locale, c'est-à-dire les arrondissements ou les municipalités faisant partie de l'agglomération montréalaise.

Pour l'échantillonnage aléatoire des sites à visiter, les carrefours du territoire d'étude ont été divisés en six groupes, selon la hiérarchie des rues présentes au carrefour. Ces six groupes sont : 1. les carrefours n'ayant que des rues de type « artère »; 2 . les carrefours ayant une rue de type « artère » et une rue de type « collectrice »; 3. les carrefours « artère-locale »; 4 . les carrefours « collectricecollectrice »; 5. les carrefours « collectrice-locale »; et 6. les carrefours formés exclusivement de rues de type « locale » (Voir figure 1 pour la répartition spatiale des sous-groupes). Pour chacun de ces six groupes, la fraction d'échantillonnage est de $5 \%$, avec un minimum de 60 intersections par strate afin de s'assurer d'avoir un nombre suffisant de carrefours dans chacun des groupes.

Les sites sélectionnés ont par la suite été visités par une des trois équipes constituées de deux observateurs, ceux-ci ayant à remplir sur place une grille d'observation préétablie comportant une pluralité de variables (items) à observer pour chaque approche [pour plus de détails sur l'enquête, voir 48]. Les items sélectionnés l'ont été en raison de leur association possible avec la sécurité des piétons et de la possibilité de les observer en une seule visite, à toute heure du jour (ce qui excluait les questions d'éclairage, notamment). Après exclusion des cas problématiques post-terrain $(\mathrm{n}=83$; ruelles, cul-de-sac et croissants et routes en réfection) et agrégation des approches, c'est-à-dire que les caractéristiques des approches ont été attribuées au carrefour, 505 carrefours ont été retenus aux fins de l'analyse (voir figure 1). La compilation des items de cette grille d'observation représente la source principale de données pour les variables proximales retenues. 


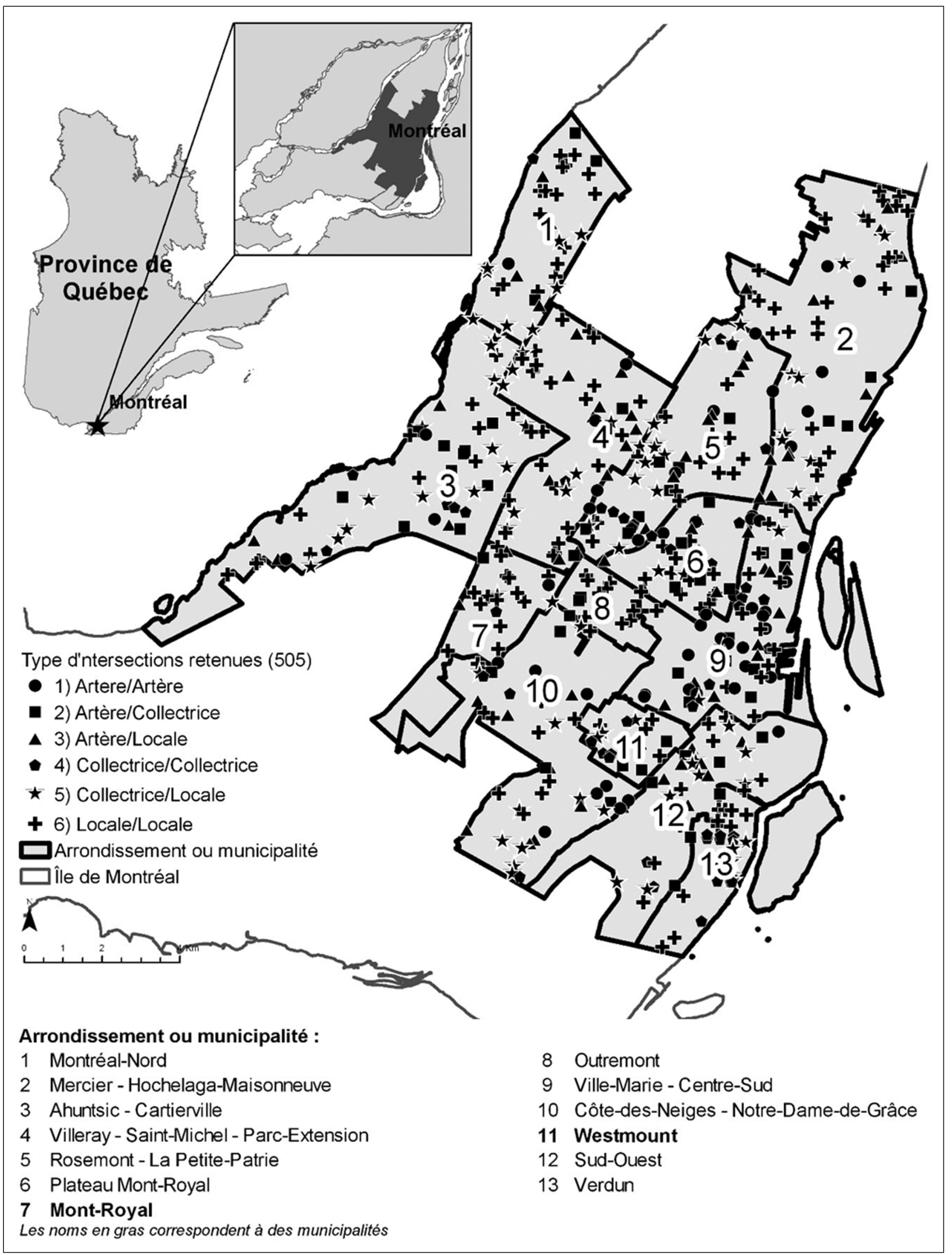

Fig. 1 Territoire d'étude et distribution spatiale des carrefours retenues $(\mathrm{n}=505)$ 
L'enquête terrain a fourni une quantité importante de données et seulement quelques variables dichotomiques ${ }^{2}$ ont été retenues dans cette étude, les plus importantes dans des analyses descriptives effectuées précédemment [45] : présence au carrefour : 1 . d'une artère, 2) de plus de cinq voies, 3) d'un feu de circulation (trois couleurs), 4) d'un feu pour les piétons, 5) d'un passage pour les piétons, 6) d'un terre-plein et 7) de mesures d'apaisement de la circulation. Finalement, rappelons que l'évaluation de la fiabilité de la grille d'observation et des mesures prises par les observateurs a été effectuée selon le principe de l'accord inter-juge [49], avec des résultats satisfaisants pour la quasi-totalité des items : fort taux de concordance et valeur du coefficient de Kappa au-dessus de 0,9, un « accord presque parfait » selon les termes de Landis et Koch [50].

Les variables distales proviennent de la mise en commun de trois bases de données dans un système d'information géographique. Avant toute chose, des zones d'influence d'un kilomètre de rayon selon la distance dans le réseau routier ont été créées autour des carrefours retenus. Ces zones ont un double objectif : de rendre compte de l'activité dans un périmètre relativement restreint autour des sites visités (c.-à-d. à « proximité »), et de créer une entité spatiale commune pour pallier à la multitude de sources et d'échelles géographiques présentes dans les données environnementales d'origine. Il est à noter qu'il n'y a pas de consensus clair sur la distance à utiliser lorsqu'il est question de «proximité », mais plusieurs études sur l'accessibilité et le potentiel piétonnier utilisent la mesure d'un kilomètre, soit 15 minutes à pied, celle que nous avons aussi retenue $[35,40,51,52]$. Par ailleurs, la création de ces zones d'influence en fonction du réseau routier a été préférée à celles de zones circulaires (rayon calculé à vol d'oiseau) puisque cette approche génère des résultats qui reflètent mieux la réalité d'un piéton qui ne marche pas en ligne droite mais bien dans un réseau de rues connectées. La figure 2 illustre par un exemple concret la mise en place de ces différentes variables à l'échelle d'une zone d'influence.

Le réseau routier utilisé pour l'échantillonnage a servi au calcul des deux premières variables distales : la longueur d'artères (en $\mathrm{km}$ ) et la mesure de connexité [46]. Pour chacune des zones d'influence, le nombre de kilomètres d'artères principales et secondaires a été extrait en faisant la somme des longueurs des tronçons redécoupés par zones. Dans la base de données du réseau routier, les artères séparées par un terre-plein central étaient représentées par deux lignes. Dans ces cas, les deux lignes ont été comptabilisées, ce qui surestime possiblement la longueur des artères pour ces cas précis. La densité de nœuds par

\footnotetext{
2 Pour les variables dichotomiques, la valeur « 0 » représente l'absence de la caractéristique et la valeur « $1 »$ représente la présence de celle-ci.
}

$\mathrm{km}^{2}$, a été choisie comme variable de connexité [34-37, 40]. À chaque fois qu'il y avait un croisement entre deux voies de circulation du réseau routier (en excluant les autoroutes, non utilisées par les piétons), un nœud était créé dans la base de données. La somme de ces nœuds par zone d'influence a permis de calculer la densité en tenant compte de l'aire totale de chacune des zones.

Le recensement de 2006 a ensuite été mis à profit pour calculer la densité de population et le nombre d'emplois brut par zone [53]. De façon à refléter plus spécifiquement la part de chaque aire de diffusion (AD : population) et secteur de recensement (SR : emplois) dans ces deux mesures, la proportion de la superficie de chacune des aires d'origine (AD ou SR) contenue dans les zones d'influence a été calculée et a servi à pondérer les données d'origine. La somme de ces données pondérées a ensuite été effectuée pour obtenir un nombre d'habitants (ensuite intégré dans le calcul de densité) ou d'emplois brut par zone d'influence, la même formule ayant été utilisée dans les deux cas (Équation 1). Une transformation logarithmique a été effectuée sur la variable finale du nombre d'emplois avant de l'intégrer aux modèles de régression en raison d'une distribution fortement anormale.

$$
E i=\sum_{j} \frac{A j}{A j i} \times E j
$$

avec $R_{\mathrm{i}}$ est le nombre d'emplois ou le nombre d'habitants dans la zone d'influence $i ; A_{\mathrm{ij}}$ est la superficie du SR ou de l'AD $j$ compris à l'intérieur de la zone d'influence $i ; A_{j}$ est la superficie totale du SR ou de l'AD $j ; R_{\mathrm{j}}$ est le nombre d'emplois du SR $j$ ou le nombre d'habitants de l'AD $j$.

Finalement, le plan d'occupation du sol le plus récent disponible (2001) a été utilisé pour calculer un indice d'entropie (mixité des usages), en tenant compte seulement de quatre types d'usages (sur un total de 16) : habitation de haute densité ; commerce de détail et centre commercial ; édifice à bureau ; équipement et service communautaire [54]. Après l'agrégation de la superficie de chacune des occupations du sol retenues par zone d'influence, nous avons calculé un indice d'entropie en nous basant sur la méthode de Frank et al. [40 : Équation 2]. Cet indice est repris dans plusieurs études, mais avec des variantes au niveau des occupations du sol choisies [34, 35, 52] :

Où $E_{i}$ est l'indice d'entropie finale par zone d'influence ; $\mathrm{n}$ est le nombre d'occupations du sol dont la superficie est plus grande que $0 ; a$ est la superficie totale couverte par les quatre occupations du sol retenues; $x_{1}$ à $x_{4}$ représente respectivement les superficies des occupations du sol suivantes : habitation de haute densité, commerce de détail et centre commercial, édifice à bureaux et équipement et service communautaire. 

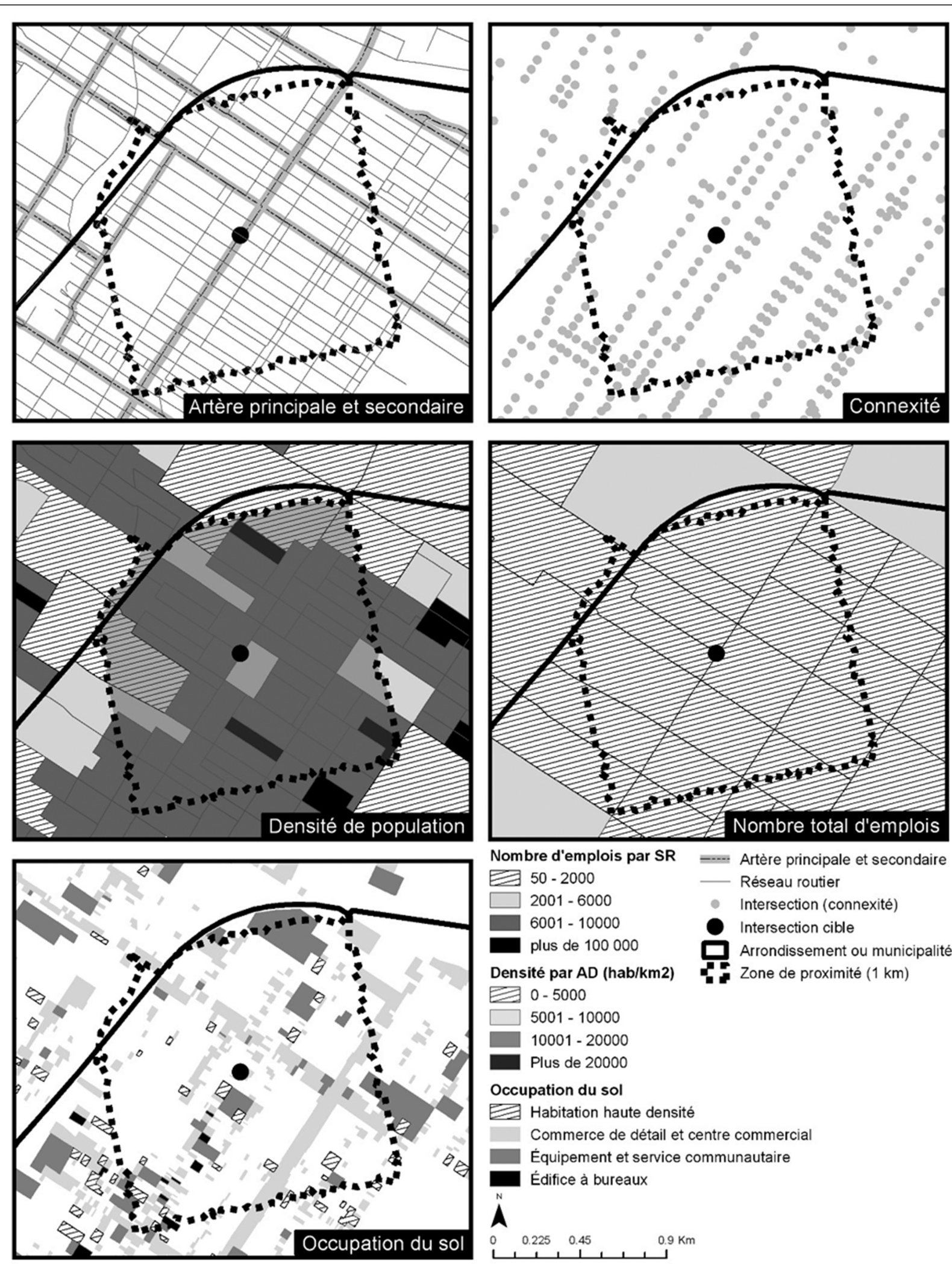

Nombre d'emplois par SR =--.. Artère principale et secondaire

ॠ0-2000

$\square 2001-6000$

$6001-10000$

- Réseau routier

plus de 100000

Densité par AD (hab/km2)

- Intersection (connexité)

$0-5000$

$\square 5001-10000$

$10001-20000$

Plus de 20000

Occupation du sol

Habitation haute densité

Commerce de détail et centre commercial

Équipement et service communautaire

Édifice à bureaux

$\hat{A}^{N}$

$\begin{array}{llll}0 & 0.225 & 0.45 \quad 0.9 & \mathrm{~km}\end{array}$

Arrondissement ou municipalité
Zone de proximité $(1 \mathrm{~km})$

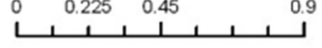

Fig. 2 Illustration de la méthode pour la création de la base de données spatiale à l'échelle des zones d'influence 
La dernière source de données mise à profit dans notre analyse est constituée des informations sur les accidents piétons, qui proviennent des systèmes d'information d'Urgences-Santé, la seule corporation ambulancière sur le territoire à l'étude [55]. Les blessés compris dans cette base de données sont caractérisés par plusieurs attributs, dont les plus intéressants dans le contexte de notre étude sont les coordonnées $X$ et $Y$ du lieu de l'intervention ambulancière et le type d'usager de la route impliqué (automobiliste, piéton, cycliste ou motocycliste). Il est à noter que la base de données inclut tous les blessés, peu importe leur gravité et inclut les décès aussi. Cette notion de gravité n'a par contre pas été étudiée ici puisque nous nous intéressons au fardeau des blessures dans leur entièreté, et non uniquement dans le cas des blessures graves ou mortelles, comme c'est fréquemment le cas dans la littérature. À partir d'une extraction des données pour la période du $1^{\text {er }}$ janvier 1999 au 31 juillet 2008, les lieux d'interventions avec au moins un blessé piéton ont été cartographiés à l'aide d'ArcGIS [56] et seuls ceux localisés dans un rayon de 50 mètres des carrefours visités ont été retenus. Un total de 781 victimes a ainsi été utilisé aux fins d'analyse $(10 \%$ du nombre total de blessés piétons recensés pour la même période dans les 13 quartiers). Ainsi, le nombre total de blessés piétons par carrefour constitue la variable dépendante utilisée pour le premier modèle d'analyse (régression binomiale négative). Le redécoupage de cette variable en trois catégories a permis de l'utiliser pour le second modèle d'analyse (régression logistique ordinale). Cette nouvelle variable comprend les modalités suivantes : 0 pour les carrefours exempts d'accidents $(\mathrm{n}=259) ; 1$ pour des carrefours ayant 1 ou 2 accidents $(n=146)$; et 2 pour ceux ayant 3 accidents ou plus $(\mathrm{n}=100)$.

Comme mentionné en introduction, aucune mesure directe du volume de trafic n'est incluse dans nos modèles, pour différentes raisons. Premièrement, cette étude porte sur un échantillon aléatoire, représentatif, d'intersections. Or, à Montréal comme dans la majorité des grandes villes d'Amérique du Nord, les volumes de trafic estimés suite aux comptages manuels ne sont disponibles que pour les carrefours ayant des feux de circulation. Deuxièmement, cette étude vise à décrire l'association potentielle entre les aménagements aux carrefours et le nombre de piétons blessés. Or, les volumes de trafic sont étroitement liés, d'une part, aux aménagements routiers et, d'autre part, au nombre de piétons blessés aux carrefours. Étant donné l'importance du trafic dans l'occurrence d'accidents, il est probable que l'intégration de cette variable dans nos modèles prédictifs aurait pour effet de «masquer » l'effet des autres variables environnementales en agissant comme un "facteur de confusion " (confounding factor) $[57,58]$. Dans le cas présent, puisque l'objectif principal est justement de tenter de quantifier l'effet de certaines variables environnementales, elles demeurent au centre de nos analyses. Troisièmement, les analyses incluent trois variables que nous considérons des proxys les plus « directs » de l'intensité de la circulation : longueur des artères dans la zone d'influence ; présence d'une artère au carrefour; et présence d'au moins une branche avec plus de cinq voies.

Deux de ces variables proxy ont pu être comparées avec les estimés des volumes de trafic aux carrefours obtenus de la Ville de Montréal pour 203 carrefours de notre échantillon (moyenne de 26259 véhicules/jour/carrefour). Ces comparaisons démontrent que la moyenne des volumes de trafic est beaucoup plus élevée aux carrefours avec artère(s) qu'aux carrefours sans artères (respectivement 32629 et 13328 véhicules/jour, $p<0,001)$. La moyenne des volumes de trafic est aussi beaucoup plus élevée aux carrefours ayant au moins une branche avec 5 voies qu'aux autres carrefours pour lesquels les volumes de trafic sont disponibles (respectivement 38080 et 17480 véhicules/jour, $\mathrm{p}<0,001$ ). Ainsi, dans les analyses, ces variables permettent de contrôler, en partie, l'effet du volume de trafic sur le nombre de piétons blessés.

\section{Modèles de régression}

Afin d'évaluer l'influence des variables proximales et distales sur l'occurrence de collisions impliquant un piéton, nous proposons d'intégrer ces variables dans deux modèles de régression, tous deux mis en œuvre dans SAS version 9.1 [59]. Le premier modèle a pour variable dépendante le nombre total de piétons blessés au carrefour (rayon de $50 \mathrm{~m}$ ). Nous avons privilégié un modèle de régression binomial négatif en raison d'une surdispersion de cette variable et d'une forte présence de valeurs à 0 (plus de $50 \%$ des carrefours) [60], comme il est commun de le faire en accidentologie [61]. Pour le second modèle, la variable étant multinomiale (aucun accident ; 1 ou 2 ; 3 ou plus), nous avons utilisé un modèle de régression logistique ordinale de façon à mieux comprendre l'effet des variables proximales et distales sur la probabilité de passer à une catégorie supérieure d'accident [62, 63]. De façon à s'assurer de l'absence de multicolinéarité entre les variables avant de les intégrer dans les modèles, nous avons calculé les coefficients de corrélation. Les résultats étaient satisfaisants : les coefficients varient entre 0,2 et 0,6 pour les variables continues, des valeurs jugées assez faibles pour intégrer toutes les variables à nos modèles.

Les variables explicatives pour ces deux modèles ont été intégrées en trois blocs. Le premier bloc regroupe les trois variables reliées aux volumes de trafic. Le second inclut les variables proximales (5 variables) tandis que le dernier bloc renvoie aux variables distales (4 variables). 


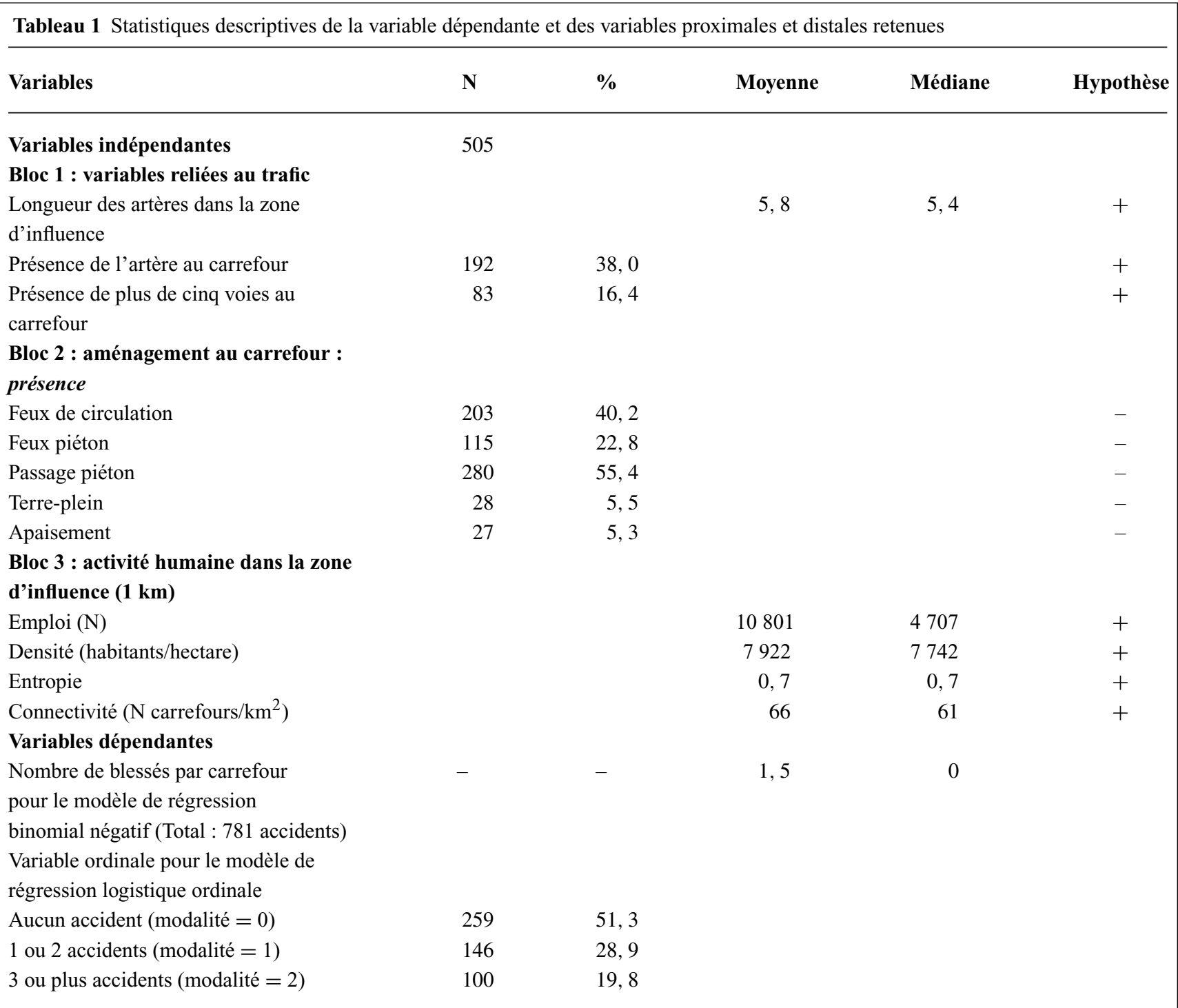

Les hypothèses initiales sur l'influence de ces variables sur la variable dépendante - augmentation ou diminution du nombre d'accidents ou de la probabilité de passer à une modalité supérieure d'accidents - étaient à la fois négatives pour le bloc 2 (variables proximales) et positives pour les blocs 1 (proxys du trafic) et 3 (variables distales). En d'autres termes, la présence accrue d'une des trois variables représentant le trafic ou d'une des quatre variables représentant un niveau plus élevé d'activité à proximité du carrefour devrait se traduire par un plus grand nombre d'accidents. À l'opposé, la présence d'éléments au carrefour visant une meilleure séparation des usagers de la route dans l'espace ou dans le temps devrait diminuer le nombre d'accidents ou la probabilité d'appartenir à une catégorie supérieure d'accidents.

La figure 3 résume la méthodologie nous permettant de répondre à l'objectif principal du présent article qui est d'évaluer l'impact relatif de variables proximales et distales sur le nombre de piétons blessés aux carrefours. Les deux premières colonnes de gauche présentent les sources de données en fonction de l'échelle d'analyse (le carrefour ou la zone d'influence), la troisième colonne détaille les variables incluses dans l'analyse et la dernière colonne rappelle les deux variables dépendantes testées selon des modèles de régression négatif-binomial et logistique ordinal.

\section{Résultats}

Le Tableau 2 présente quelques statistiques descriptives des variables intégrées aux modèles. Le premier bloc de variables regroupant les proxys au trafic rappelle l'importance de la présence des artères dans les quartiers centraux de Montréal puisque la longueur des artères dans les zones d'influence s'élève à près de $6 \mathrm{~km}$ en moyenne. 


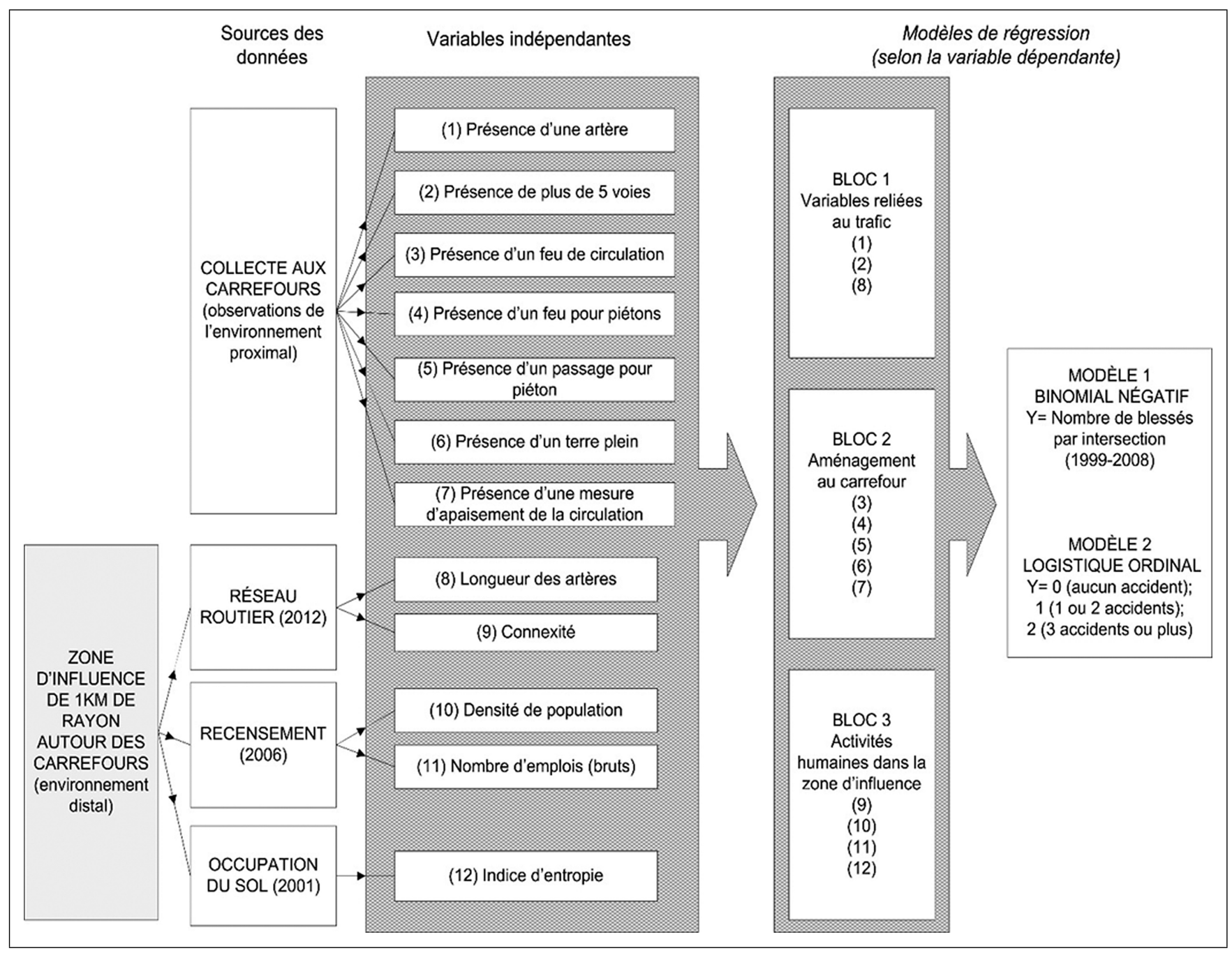

Fig. 3 Organigramme méthodologique

De plus, dans notre échantillon, il y avait une ou des artère(s) à près de $40 \%$ des carrefours et un peu plus de $16 \%$ des carrefours avaient au moins une approche avec plus de cinq voies. Le second bloc, regroupant les variables proximales, montre une grande variation dans la présence de certaines des variables : alors que seulement $5 \%$ des carrefours de l'échantillon bénéficient de mesures d'apaisement de la circulation ou d'un terre-plein, $40 \%$ ont un feu de circulation et plus de la moitié ont une traversée piétonne balisée (marquage au sol, peu importe l'état de celui-ci, récent ou quasi-effacé). Le dernier bloc regroupant les variables distales présente des moyennes et des médianes assez similaires, sauf pour l'emploi : comme mentionné précédemment, cette dernière variable n'est pas distribuée normalement en raison de la forte concentration des emplois dans certaines zones. Pour ce qui est des variables dépendantes, il y a en moyenne 1,5 accidents à moins de 50 mètres des carrefours, même si plus de la moitié des carrefours visités $(51,3 \%)$ n'ont pas eu d'accidents impliquant un piéton. Finalement, ce sont près de $20 \%$ des carrefours qui ont eu au moins 3 blessés piétons durant la période d'étude.

\section{Portrait géographique des variables à l'étude}

\section{Variables proximales : au carrefour}

La localisation des variables proximales ne semble pas nécessairement suivre une logique géographique, quoique nous puissions déjà entrevoir l'influence de certaines politiques locales d'aménagement des carrefours. Par exemple, nous constatons à la figure 4 que la présence d'artère(s), de feux de circulation et de feux pour piétons semble être plus marquée dans l'arrondissement VilleMarie, qui est constitué de certaines parties résidentielles mais qui représente essentiellement le quartier des affaires et des universités. Cependant, la présence de passage pour piéton semble distribuée plus équitablement entre les différents quartiers à l'étude. La figure 4 illustre aussi le 


\begin{tabular}{|c|c|c|c|c|c|c|c|c|c|c|c|}
\hline \multirow[t]{2}{*}{ Variables } & \multicolumn{2}{|l|}{ Modèle 1} & \multicolumn{2}{|l|}{ Modèle 2} & \multicolumn{2}{|c|}{ Modèle 3} & \multicolumn{2}{|c|}{ Modèle final } & \multirow[b]{2}{*}{$\begin{array}{l}\text { Rapport } \\
\text { de cote }\end{array}$} & \multirow{2}{*}{\multicolumn{2}{|c|}{$\begin{array}{l}\text { Intervalle } \\
\text { de } \\
\text { confiance } \\
95 \%\end{array}$}} \\
\hline & $\beta$ & $\mathbf{p}$ & $\beta$ & $\mathbf{p}$ & $\beta$ & $\mathbf{p}$ & $\beta$ & $\mathbf{p}$ & & & \\
\hline \multicolumn{11}{|c|}{ 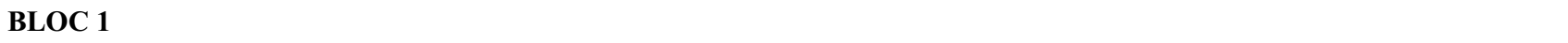 } & \\
\hline Longueur des artères & 0,000 & $<0,0001$ & 0,000 & 0,000 & 0,000 & 0,645 & & & & & \\
\hline $\begin{array}{l}\text { Présence d'une } \\
\text { artère }\end{array}$ & 0,531 & 0,002 & 0,021 & 0,896 & 0,114 & 0,457 & & & & & \\
\hline $\begin{array}{l}\text { Présence de plus de } \\
\text { cinq voies }\end{array}$ & 0,646 & 0,001 & 0,436 & 0,010 & 0,650 & $<0,0001$ & 0,673 & $<0,0001$ & 1,961 & 1,158 & 1,471 \\
\hline \multicolumn{12}{|l|}{ BLOC 2} \\
\hline Feux de circulation & & & 0,955 & $<0,0001$ & 0,953 & $<0,0001$ & 0,963 & $<0,0001$ & 2,619 & 1,218 & 1,779 \\
\hline Feux piéton & & & 0,403 & 0,013 & 0,327 & 0,034 & 0,338 & 0,025 & 1,403 & 1,163 & 1,043 \\
\hline Passage piéton & & & 0,552 & 0,006 & 0,463 & 0,020 & 0,447 & 0,023 & 1,563 & 1,218 & 1,062 \\
\hline Terre-plein & & & $-0,321$ & 0,192 & $-0,131$ & 0,580 & & & & & \\
\hline Apaisement & & & 0,232 & 0,378 & 0,153 & 0,541 & & & & & \\
\hline \multicolumn{12}{|l|}{ BLOC 3} \\
\hline Emploi (log) & & & & & 0,244 & 0,019 & 0,267 & $<0,0001$ & 1,306 & 1,060 & 1,166 \\
\hline Habitants/hectare & & & & & 0,104 & $<0,0001$ & 0,100 & $<0,0001$ & 1,105 & 1,021 & 1,062 \\
\hline Entropie & & & & & $-0,623$ & 0,117 & & & & & \\
\hline Connectivité & & & & & 0,002 & 0,570 & & & & & \\
\hline $\mathrm{AIC}$ & & 1595,220 & & 1479 & 9,366 & 1451 & 1,243 & 1443 & 3,687 & & \\
\hline
\end{tabular}

faible nombre de carrefours avec un terre-plein ou des mesures d'apaisement.

\section{Variables distales : dans la zone d'influence du carrefour visité}

Tout comme dans le cas des variables proximales, celles représentant l'environnement bâti forment un certain patron spatial partant du centre-ville, où la majorité d'entre elles sont plus importantes, vers les quartiers adjacents, puis vers les quartiers en périphérie du territoire d'étude, où leur présence est moins marquée. À des fins de simplification, les cartes ne sont pas présentées ici, mais sont disponibles dans une publication précédente qui peut être mise à la disposition des lecteurs intéressés [64]. C'est ainsi que la longueur d'artères par zone d'influence est plus importante au centre-ville, où ces artères agissent en tant que voies d'entrée et de sortie du trafic, et dans les arrondissements au nord et à l'est du centre-ville. Dans certains quartiers, les artères sont plus rares ou limitées à la frontière de l'arrondissement (ex : Mont-Royal et Outremont : [65]). Par ailleurs, le portrait est relativement homogène lorsqu'il est question de la densité des carrefours puisque la quasi-totalité du territoire d'étude est constituée de plans de rues orthogonales, maximisant leur nombre. Pour leur part, la densité de population et le nombre d'emplois ont une répartition spatiale qui diffère quelque peu, mais qui concorde avec les occupations du sol qu'on retrouve dans chacun des quartiers : un pôle d'emploi très important subsiste au centre-ville (Ville-Marie), cette zone étant caractérisée par la plus grande mixité des usages et par la présence accrue d'édifices à bureaux, mais aussi par une densité d'habitants qui n'est pas la plus élevée. Les quartiers adjacents au centre-ville ont pour leur part une présence marquée de rues commerciales, combinée avec des habitations à haute densité, directement reliées à la forte densité de population qu'on y retrouve (ex. : Plateau-MontRoyal, Mercier-Hochelaga-Maisonneuve, Côte-des-NeigesNotre-Dame-de-Grâce). Finalement, les quartiers les plus en périphérie de notre territoire d'étude se caractérisent par 


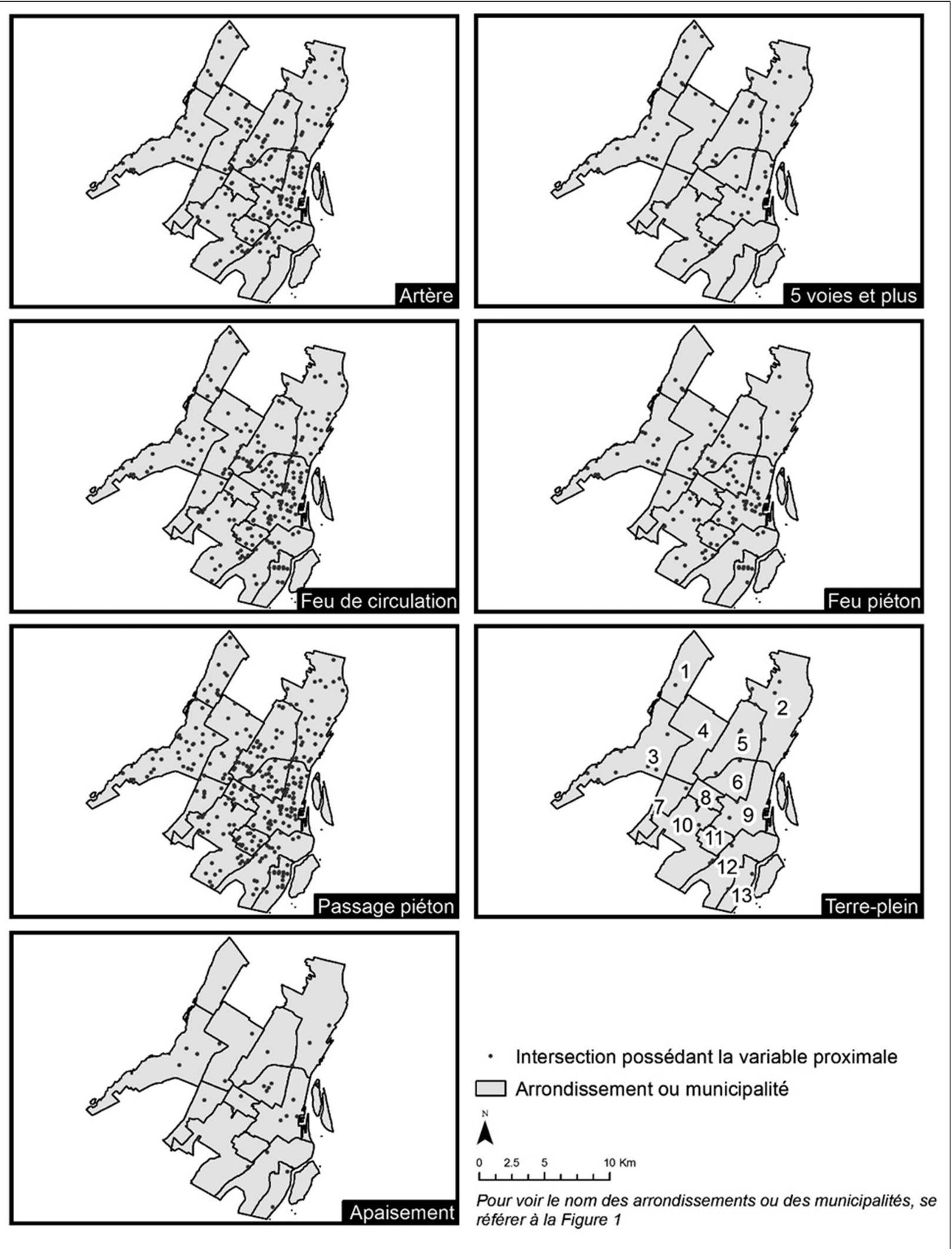

Fig. 4 Distribution géographique des variables proximales (observations au carrefour) 
une faible présence des occupations du sol retenues (donc peu de services propices aux déplacements piétons), peu d'emplois sauf pour des quartiers spécifiques (présence de parcs industriels, d'installations ferroviaires ou portuaires), et une densité de population moins importante, surtout au nord-ouest (Ahuntsic-Cartierville, Mont-Royal).

\section{Blessés piétons}

Les carrefours de notre échantillon ayant un ou plusieurs piétons blessés semblent se concentrer spatialement, notamment dans les arrondissements Ville-Marie, Plateau Mont-Royal et Rosemont - La Petite-Patrie (Figure 5).

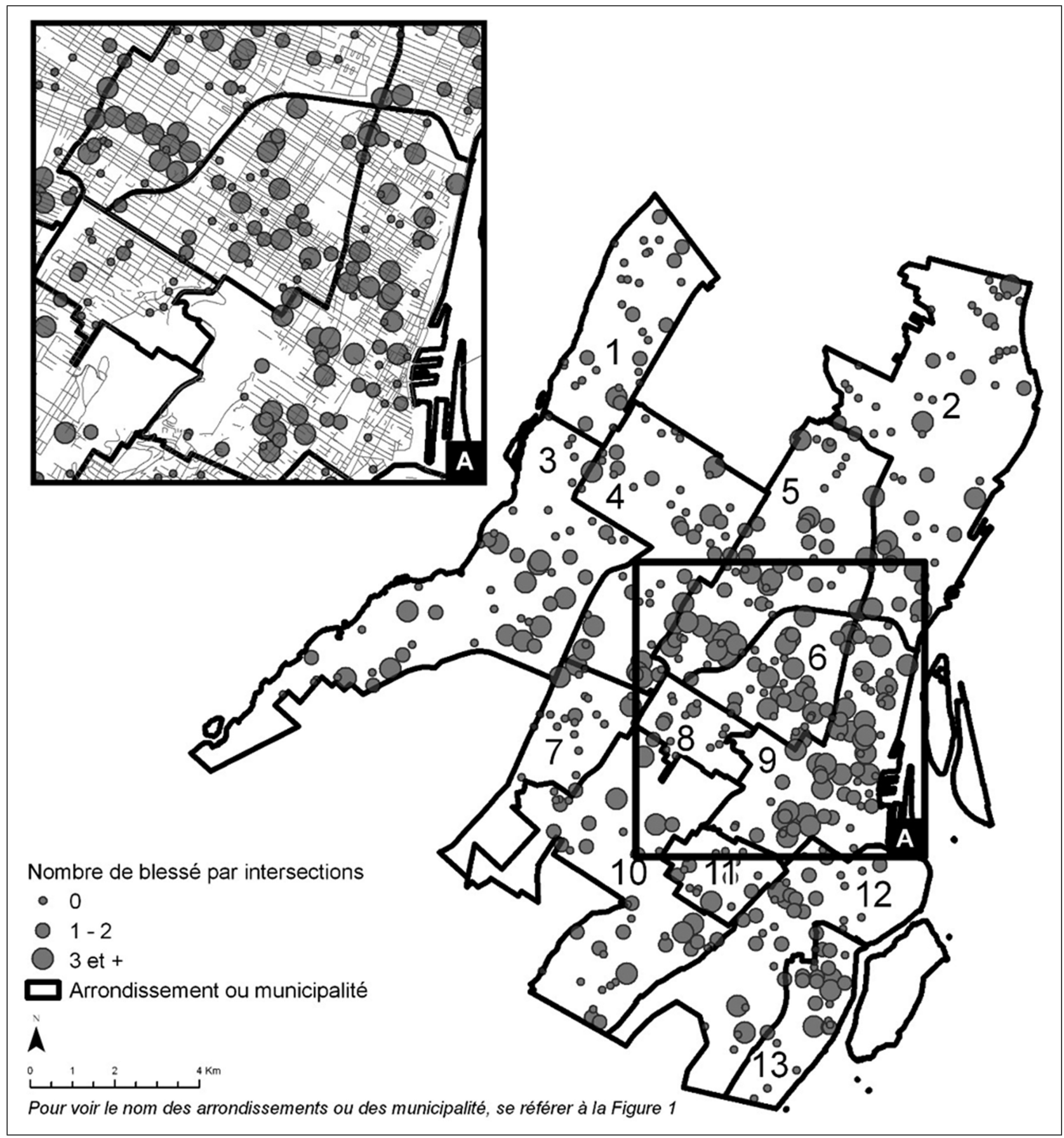

Fig. 5 Nombre de piétons blessés aux 505 carrefours étudiées (1999-2008) 


\begin{tabular}{|c|c|c|c|c|c|c|c|c|c|c|c|}
\hline \multirow[t]{2}{*}{ Variables } & \multicolumn{2}{|l|}{ Modèle 1} & \multicolumn{2}{|l|}{ Modèle 2} & \multicolumn{2}{|l|}{ Modèle 3} & \multicolumn{2}{|c|}{ Modèle final } & \multirow[b]{2}{*}{$\begin{array}{l}\text { Rapport } \\
\text { de cote }\end{array}$} & \multirow{2}{*}{\multicolumn{2}{|c|}{$\begin{array}{l}\text { Intervalle } \\
\text { de } \\
\text { confiance } \\
95 \%\end{array}$}} \\
\hline & $\beta$ & $\mathbf{p}$ & $\beta$ & $\mathbf{p}$ & $\beta$ & $\mathbf{p}$ & $\beta$ & p & & & \\
\hline Constante 0 & $-1,092$ & $<0,0001$ & $-1,674$ & $<0,0001$ & $-5,963$ & $<0,0001$ & $-5,775$ & $<0,0001$ & & & \\
\hline $\begin{array}{l}\text { Constante } 1 \\
\text { BLOC } 1\end{array}$ & $-2,680$ & $<0,0001$ & $-3,585$ & $<0,0001$ & $-8,004$ & $<0,0001$ & $-7,796$ & $<0,0001$ & & & \\
\hline Longueur des artères & 0,000 & 0,001 & 0,000 & 0,003 & 0,000 & 0,830 & & & & & \\
\hline $\begin{array}{l}\text { Présence d'une } \\
\text { artère }\end{array}$ & 0,992 & $<0,0001$ & 0,232 & 0,337 & 0,465 & 0,065 & 0,436 & 0,062 & 1,546 & 0,979 & 2,443 \\
\hline $\begin{array}{l}\text { Présence de plus de } \\
\text { cinq voies } \\
\text { BLOC } 2\end{array}$ & 0,896 & 0,000 & 0,812 & 0,005 & 1,071 & 0,001 & 1,043 & 0,000 & 2,838 & 1,638 & 4,918 \\
\hline Feux de circulation & & & 1,227 & $<0,0001$ & 1,338 & $<0,0001$ & 1,570 & $<0,0001$ & 4,808 & 2,901 & 7,968 \\
\hline Feux piéton & & & 0,789 & 0,004 & 0,678 & 0,015 & 0,689 & 0,013 & 1,991 & 1,158 & 3,423 \\
\hline Passage piéton & & & 0,536 & 0,050 & 0,358 & 0,215 & & & & & \\
\hline Terre-plein & & & $-0,631$ & 0,134 & $-0,488$ & 0,259 & & & & & \\
\hline Apaisement & & & 0,517 & 0,203 & 0,465 & 0,265 & & & & & \\
\hline BLOC 3 & & & & & & & & & & & \\
\hline Emploi (log) & & & & & 0,477 & 0,006 & 0,375 & 0,000 & 1,455 & 1,191 & 1,777 \\
\hline Habitants / hectare & & & & & 0,000 & $<0,0001$ & 0,183 & $<0,0001$ & 1,201 & 1,125 & 1,283 \\
\hline Entropie & & & & & $-0,792$ & 0,222 & & & & & \\
\hline Connectivité & & & & & $-0,006$ & 0,286 & & & & & \\
\hline AIC & & & 946,000 & & 854,000 & 819,000 & 813,549 & & & & \\
\hline Pseudo- $\mathrm{R}^{2}$ & & & 0,199 & & 0,372 & 0,436 & 0,427 & & & & \\
\hline
\end{tabular}

Ce constat n'est pas étonnant au regard des publications existantes [55], et s'explique par une combinaison de facteurs incluant le trafic mais aussi la présence des variables distales telles que décrites précédemment. Par ailleurs, la figure 5 démontre aussi une certaine dispersion des carrefours avec blessés sur le territoire à l'étude, puisqu'il $\mathrm{y}$ en a dans presque tous les quartiers.

\section{Modèles}

\section{Modèle négatif binomial sur la fréquence de blessés piétons aux carrefours}

Le Tableau 3 présente les résultats des différents modèles calculés avec comme variable dépendante le nombre de blessés piétons aux carrefours : on peut y voir une amélioration de $\mathrm{l}^{\prime} \mathrm{AIC} \mathrm{C}^{3}$ d'un modèle à l'autre, après l'intégration de chacun des blocs de variables thématiques, ce qui suggère que le dernier modèle offre une meilleure estimation des paramètres. Le modèle final pour lequel uniquement les variables significatives ont été conservées confirme la significativité d'au moins une variable dans chacun des blocs. C'est ainsi que la présence de plus de 5 voies, la présence d'un feu de circulation, d'un feu piéton et d'un passage pour piéton, augmentent le risque de blessés piétons, tout comme le nombre d'emplois et la densité de population. Les rapports de cote nous permettent d'affirmer que la probabilité d'une collision impliquant un piéton est 2,6 fois plus élevée aux carrefours ayant un feu de circulation

\footnotetext{
${ }^{3}$ AIC : Le critère d'information d'Akaike est une mesure de la qualité d'un modèle statistique. Plus il est faible, meilleur est le modèle.
} 
et près de 2 fois plus élevée aux carrefours ayant plus de cinq voies. Ces probabilités sont aussi $40 \%$ plus élevées s'il y a un feu piéton et $56 \%$ plus élevées aux carrefours avec passage(s) pour piéton. En ce qui concerne l'environnement entourant les carrefours, la densité d'emploi et la densité de population sont tous deux associés à une augmentation de la probabilité de collisions impliquant un piéton. Ces premiers résultats tendent à confirmer nos hypothèses initiales pour les proxys du trafic (bloc 1) et les variables distales (bloc 3) mais tendent à infirmer nos hypothèses initiales pour les variables proximales liées aux aménagements des carrefours (bloc 2) ; nous y reviendrons en discussion.

\section{Modèle logistique ordinal}

Le Tableau 3 présente les résultats des différents modèles sur les sous-groupes de carrefours divisés selon leur nombre d'accidents. Ce modèle logistique ordinal calcule la probabilité de passer d'une catégorie de blessés à celle supérieure en fonction des variables insérées dans le modèle en trois blocs. Comme dans les modèles précédents, nous observons une amélioration de l'AIC à chaque intégration d'un nouveau bloc, tout comme le pseudo R-carré qui passe de 0,2 à 0,43 , un résultat tout à fait acceptable dans la mesure où nous avons utilisé des variables indirectes pour mesurer le trafic. Les variables significatives sont presque les mêmes que dans les modèles précédents, avec quelques exceptions : la présence d'un passage piéton n'est plus statistiquement significative, tandis que la présence d'une artère au carrefour l'est, à la marge $(\mathrm{p}=0,06)$, contrairement au premier modèle (négatif binomial). C'est ainsi que la présence d'un feu de circulation fait augmenter de près de 5 fois les chances de se retrouver dans une catégorie avec un nombre de blessés plus élevé. La présence d'un feu pour piétons fait augmenter cette probabilité de 2 fois, de 1,5 fois lorsqu'il $\mathrm{y}$ a une artère et de près de 3 fois lorsqu'il y a plus de 5 voies sur au moins une approche. Pour ce qui est des variables distales, ce sont les mêmes variables que dans le modèle précédent qui augmentent la probabilité de passer à une catégorie supérieure d'accident, soit la densité d'emploi (rapport de cote de 1,5) et la densité de population (rapport de cote de 1,2).

\section{Discussion}

L'objectif principal de cet article était d'explorer l'influence de variables d'aménagement et d'occupation du sol sur le risque d'accident piéton à partir de données recueillies pour un échantillon représentatif de carrefours des quartiers centraux de Montréal. Les deux modèles testés ont permis de démontrer l'influence de plusieurs variables reliées à l'aménagement des carrefours et à l'activité piétonne et véhiculaire autour de ces mêmes carrefours. Contrairement à nos hypothèses de départ qui affirmaient que les mécanismes présents aux carrefours pour réguler les interactions entre les différents usagers de la route (feux, passage piéton, etc.) réduiraient le risque piéton, il semble que les variables que nous avons introduites dans nos modèles sont toutes reliées à un plus grand nombre d'accidents impliquant un blessé piéton, sauf pour la présence de terre-plein, qui n'est cependant pas statistiquement significative. L'effet « protecteur » souhaité des aménagements ne semble donc pas assez important pour réduire le risque d'accidents impliquant des piétons en milieu urbain aux intersections qui en ont le plus besoin. Pour ce qui est des variables « distales », nos résultats mettent en avant l'importance des pôles d'emplois dans le risque piéton. Ce résultat confirme l'importance potentielle des différents générateurs de déplacement dans la ville, en particulier lorsque ceux-ci génèrent à la fois des volumes piétons et véhiculaires : institutions d'éducation (écoles, universités), stations intermodales (gare, métro, terminus d'autobus), etc.

Nos résultats ne sont pas sans rappeler que nos villes, en particulier dans le contexte nord-américain dans lequel se retrouve Montréal, sont encore le reflet d'un développement urbain et d'aménagements routiers priorisant l'automobile [27, 66-68]. La configuration actuelle de la vaste majorité $\mathrm{du}$ réseau routier est régie par une nécessaire fluidité des déplacements induisant la pratique de vitesse parfois excessive en regard de la pratique de modes de transports dits « doux » (marche, vélo) [69]. Le piéton doit s'ajuster à cette réalité en se déplaçant dans un environnement physique qui n'est pas toujours adapté ni sécuritaire, mais qui semble plutôt contribuer, du moins en partie, à l'augmentation du risque de collision et de blessures.

Les arrondissements et les municipalités de notre territoire d'étude ont tous une responsabilité partagée quant à la voirie et à la signalisation routière : ce partage est fait en fonction de la hiérarchie routière, donnant juridiction au Ministère des Transports du Québec dans le cas des routes nationales et autoroutes, à la Ville-centre ${ }^{4}$ dans le cas des artères et de certaines collectrices et aux arrondissements et municipalités pour le reste du réseau local. Conséquemment, ces territoires ont été développés dans des contextes sociaux, économiques et historiques différents. Par exemple, Ville-Marie, qui est l'arrondissement où il y a eu le plus d'accidents dans la période retenue (1 532 blessés, soit $21 \%$ du total pour l'île de Montréal), est un lieu de destination pour des milliers de travailleurs quotidiennement. Dès 1950 « l'automobile impose ses énormes exigences. Comme les

\footnotetext{
${ }^{4}$ Malgré la fusion de plusieurs anciennes villes sur le territoire de l'île de Montréal, il existe toujours un partage des pouvoirs entre l'administration centrale et les arrondissements.
} 
pièces d'un immense casse-tête, on agence les multiples parcelles de l'ancien cadastre pour faire place aux superstructures du centre-ville» [50]. À cette époque, de grands projets d'infrastructure sont réalisés tels que l'échangeur Turcot (1967) et l'élargissement du boulevard Dorchester (1954-1955 : maintenant appelé René-Lévesque), pour contenir le flot grandissant d'automobiles [51]. À l'heure où des parties importantes de ce réseau sont en réfection, les autorités montréalaises et québécoises ont devant eux une opportunité de remettre en question ce modèle centré sur l'utilisation de la voiture en solo, et de proposer, du moins dans certains quartiers centraux où la trame urbaine le permet déjà, une certaine réappropriation de la rue par les transports collectifs et les piétons : celle-ci peut et doit être considérée comme un espace public au sens où Jane Jacob l'entendait [52, 53].

C'est dans ce cadre que certaines initiatives locales récentes se traduisent par de réels changements dans la trame urbaine. L'arrondissement du Plateau Mont-Royal fait office de modèle en ce sens puisqu'il a été le premier arrondissement à adopter un plan de déplacement urbain en 2009. Ce plan a pour objectif de « mieux partager l'espace urbain et la voirie pour améliorer la cohabitation de tous les utilisateurs » et de "redéfinir la place des différents modes de transport dans l'espace urbain » [70]. La démarche entreprise par cet arrondissement repose justement sur la consultation d'un grand nombre de partenaires locaux et sur l'élaboration d'un plan d'action incluant le réaménagement de carrefours ou encore l'implantation de mesures d'apaisement de la circulation, par exemple aux abords des écoles [71]. De telles initiatives devraient permettre de revoir le rôle des différents éléments présents aux carrefours et de leur effet sur la sécurité des piétons.

\section{Conclusion}

À partir de deux modèles de régression prédictifs, nous avons pu répondre à notre objectif principal qui était d'établir si des caractéristiques de l'environnement proximal et distal des carrefours pouvaient avoir un effet sur le nombre de blessés piétons. Nos hypothèses sur les variables distales telles que la densité d'emploi et de population ont été confirmées. Cependant, nos hypothèses sur une éventuelle relation négative entre les aménagements aux carrefours et les blessés piétons n'ont pas été confirmées : aucun aménagement mesuré ne semble réduire le nombre de blessés aux carrefours des quartiers centraux de Montréal.

Nos travaux comportent toutefois quelques lacunes qu'il est important de souligner. Comme nous l'avons déjà mentionné, notre prise en considération du trafic s'est faite indirectement, à défaut d'avoir des données de débits disponibles à tous les carrefours de notre échantillon. Des données plus précises sur les flux de véhicules et de piétons permettraient de mieux distinguer le rôle spécifique de chaque facteur : volumes de véhicules et de piétons, aménagements routiers, environnement urbain. Par ailleurs, nous avons traité les aménagements aux carrefours comme étant tous « égaux » en ne rapportant que leur présence ou absence, bien qu'il semble faire consensus que le phasage des feux, par exemple, peut être le révélateur d'une configuration beaucoup plus complexe. En ce sens, la prise en compte de plusieurs éléments (feux, phasage, nombre de voies, sens de circulation, flux entrants et sortants, etc.) dans une certaine « catégorisation » de ces carrefours rendrait plus exacte le portrait de l'influence de ces aménagements sur le risque routier. Nous sommes par contre d'avis que cela ne changerait pas les conclusions générales de nos travaux, affirmant que le piéton nécessite une plus grande considération lorsqu'il est question d'aménagement aux carrefours, le lieu de rencontre unique et incontournables de tous les usagers de la route.

\section{Références}

1. Organisation mondiale de la Santé (2004) Rapport mondial sur la prévention des traumatismes dus aux accidents de la circulation. OMS, Genève, $210 \mathrm{p}$.

2. Mayou R, Bryant B (2003) Consequences of road traffic accidents for different types of road user. Injury 34: 197-202.

3. Société de l'assurance automobile du Québec (2010) Bilan 2009 : accidents, parc automobile et permis de conduire. Direction des études et des stratégies en sécurité routière, Québec, 213 p.

4. Guillerme A (2008). La mise au pas du piéton à Paris au temps de l'autophilie (1900-1930). In : Granié M.-A., Auberlet J.-M. (éds). Le piéton et son environnement. Quelles interactions? Quelles adaptations? Institut National de Recherche sur les Transports et leur Sécurité, Paris, pp 33-45.

5. Forum international des transports (2012) Piétons : Sécurité, espace urbain et santé. Éditions OCDE, $121 \mathrm{p}$.

6. Transports Canada (2004) La sécurité des usagers de la route vulnérables : Un enjeu mondial. Ottawa, $9 \mathrm{p}$.

7. Julien A, Carré J-R (2002) Cheminements piétonniers et exposition au risque. Recherche Transports Sécurité 76 : 173-189.

8. Black W R (2010) Sustainable transportation: problems and solutions. Guilford Press, New York, 299 p.

9. Wang C, Quddus M A, Ison S G (2013) The effect of traffic and road characteristics on road safety: A review and future research direction. Safety Science 57: 264-75.

10. Shinar D (2012) Safety and mobility of vulnerable road users: Pedestrians, bicyclists, and motorcyclists. Accid Anal Prev 44: 1-2.

11. Pulugurtha S S, Repaka S R (2008) Assessment of models to measure pedestrian activity at signalized intersections. Transport Res Rec 2073: 39-48.

12. Retting R, Ferguson S, McCartt A (2003) A review of evidencebased traffic engineering measures designed to reduce pedestrianmotor vehicle crashes. Am J Public Health 93: 1456-63.

13. Rifaat S, Tay R, De Barros A (2011) Effect of street pattern on the severity of crashes involving vulnerable road users. Accid Anal Prev 43: 276-83.

14. Persaud B, Hauer E, Retting R, et al. (1997) Crash reductions related to traffic signal removal in Philadelphia. Accid Anal Prev 29: $803-10$.

15. Pulugurtha S, Desai A, Pulugurtha N (2010) Are pedestrian countdown signals effective in reducing crashes? Traffic Inj Prev 11: $632-41$. 
16. Zegeer C V, Stewart J R, Huang H H, et al. (2005) Safety Effects of Marked versus Unmarked Crosswalks at Uncontrolled Locations: Final Report and Recommended Guidelines. Federal Highway Administration, $114 \mathrm{p}$.

17. Koepsell T, McCloskey L, Wolf M, et al. (2002) Crosswalk markings and the risk of pedestrian-motor vehicle collisions in older pedestrians. JAMA 288: 2136-43.

18. Leden L, Wikström P E, Gårder P, et al. (2006) Safety and accessibility effects of code modifications and traffic calming of an arterial road. Accid Anal Prev 38: 455-61.

19. Gårder P E (2004) The impact of speed and other variables on pedestrian safety in Maine. Accid Anal Prev 36: 533-42.

20. Lee C, Abdel-Aty M (2005) Comprehensive analysis of vehiclepedestrian crashes at intersections in Florida. Accid Anal Prev 37: 775-86.

21. Torbic D J, Harwood D W, Bokenkroger C D, et al. (2010) Pedestrian safety prediction methodology for urban signalized intersections. Transport Res Rec 2198: 65-74.

22. Morency P, Archambault J, Cloutier M-S, et al. (2013) Sécurité des piétons en milieu urbain: enquête sur les aménagements routiers aux intersections. Direction de santé publique, Agence de la santé et des services sociaux, Montréal, $46 \mathrm{p}$.

23. Bellefleur O, Gagnon F (2012) Apaisement de la circulation urbaine et santé: revue de littérature. Centre de collaboration nationale sur les politiques publiques et la santé, Québec, 148 p.

24. Zein S R, Geddes E, Hemsing S, et al. (1997) Safety benefits of traffic calming. Transport Res Rec 1578: 3-10.

25. Grayling T, Hallam K, Graham D, et al. (2002) Streets Ahead, Safe and Liveable Streets for Children. Institute for Public Policy Research, Londres, $32 \mathrm{p}$.

26. Transport Québec (2011) La modération de la circulation: Fiche d'information technique. Direction de la sécurité en transport: Service de l'expertise et du soutien technique en sécurité, Québec, $4 \mathrm{p}$.

27. Dumbaugh E, Li W (2011) Designing for the safety of pedestrians, cyclists, and motorists in urban environments. J Am Plann Assoc 77: 69-88.

28. Lightstone A S, Dhillon P K, Peek-Asa C, et al. (2001) A geographic analysis of motor vehicle collisions with child pedestrians in Long Beach, California: Comparing intersection and midblock incident locations. Inj Prev 7: 155-60.

29. Schuurman N, Cinnamon J, Crooks V A, et al. (2009) Pedestrian injury and the built environment: An environmental scan of hotspots. BMC Public Health 9: 1-10 (en ligne).

30. Morency P, Gauvin L, Plante C, et al. (2012) Neighborhood social inequalities in road traffic injuries: The influence of traffic volume and road design. Am J Public Health 102: 1112-9.

31. Morency P, Gauvin L, Tessier F, et al. (2011) Analyse désagrégée des facteurs environnementaux associés au nombre d'enfants blessés par un véhicule à moteur en milieu urbain. Cahiers de géographie du Québec 55: 449-68.

32. Cloutier M S, Apparicio P (2010) L'environnement autour des écoles a-t-il un impact sur le risque routier impliquant des enfants piétons à Montréal? Apport de la régression de Poisson géographiquement pondérée. Territoire en Mouvement 2008(01) : 25-38.

33. Gourvil L, Joubert F (2004) Évaluation de la congestion routière dans la région de Montréal. Ministère des Transports, Québec, $123 \mathrm{p}$.

34. Brown B, Yamada I, Smith K, et al. (2009) Mixed land use and walkability: Variations in land use measures and relationships with BMI, overweight, and obesity. Health and Place 15: $1130-41$.

35. Dygryn J, Mitas J, Stelzer J (2010) The influence of built environment on walkability using geographic information system. J Hum Kinet 24: 93-9.
36. Leslie E, Coffee N, Frank L, et al. (2007) Walkability of local communities: Using geographic information systems to objectively assess relevant environmental attributes. Health and Place 13: 111-22.

37. Smith K R, Brown B B, Yamada I, et al. (2008) Walkability and Body Mass Index. Density, Design, and New Diversity Measures. Am J Prev Med 35: 237-44.

38. Girling C, Kellett R (2005) Skinny Streets \& Green Neighborhoods. Island Press, Washington, $173 \mathrm{p}$.

39. Riva M, Apparicio P, Gauvin L, et al. (2008) Establishing the soundness of administrative spatial units for operationalising the active living potential of residential environments: An exemplar for designing optimal zones. Int $J$ Health Geogr 7: 1-13 (en ligne).

40. Frank L D, Schmid T L, Sallis J F, et al. (2005) Linking objectively measured physical activity with objectively measured urban form: Findings from SMARTRAQ. Am J Prev Med 28: 117-25.

41. Saelens B, Handy S (2008) Built environment correlates of walking: a review. Med Sci Sport Exer 40: S550-66.

42. Pulugurtha S, Repaka S (2008) Assessment of models to measure pedestrian activity at signalized intersections. Transport Res Rec 2073: 39-48.

43. Wier M, Weintraub J, Humphreys E H, et al. (2009) An area-level model of vehicle-pedestrian injury collisions with implications for land use and transportation planning. Accid Anal Prev 41: 137-45.

44. Moudon A V, Lin L, Jiao J, et al. (2011) The risk of pedestrian injury and fatality in collisions with motor vehicles, a social ecological study of state routes and city streets in King County, Washington. Accid Anal Prev 43: 11-24.

45. Cloutier M S, Tremblay M, Morency P (2013). Aménager les intersections en milieu urbain : quelle place pour le piéton? In: Actes du 3e Colloque International du GERI COPIE 2011 : Qualité et sécurité du déplacement piéton : facteurs, enjeux et nouvelles actions. Paris, pp 21-34.

46. Ville de Montréal (2002) Géobase du réseau routier de l'île de Montréal. Direction des services géomatiques, Montréal.

47. Niro F. 2007. Assemblée publique sur la révision du réseau artériel de Montréal : Présentation à la Commission permanente du conseil municipal sur le transport, la gestion des infrastructures et l'environnement. Service des infrastructures, du transport et de l'environnement, Montréal.

48. Tremblay M (2010) Validation d'une enquête terrain et analyses des intersections en fonction de leurs aménagements pour les piétons. Rapport de stage de maîtrise, Institut national de la recherche scientifique et Direction de santé publique, Montréal, $69 \mathrm{p}$.

49. Paquet C, Cargo M, Kestens Y, et al. (2010) Reliability of an instrument for direct observation of urban neighbourhoods. Landscape Urban Plan 97: 194-201.

50. Landis J, Koch G (1977) The Measurement of Observer Agreement for Categorical Data. Biometrics 33: 159-74.

51. Oliver L, Schuurman N, Hall A (2007) Comparing circular and network buffers to examine the influence of land use on walking for leisure and errands. Int J Health Geogr 6: 1-11 (en ligne).

52. Riva M, Gauvin L, Apparicio P, et al. (2009) Disentangling the relative influence of built and socioeconomic environments on walking: The contribution of areas homogenous along exposures of interest. So Sci Med 69: 1296-305.

53. Canada Statistique (2006) Recensement de la population, Ottawa.

54. Communauté urbaine de Montréal (CUM) (2001) Carte d'occupation du sol. Division de l'aménagement, service de la mise en valeur du territoire, Montréal.

55. Morency P, Cloutier M S (2005) Distribution géographique des blessés de la route sur l'île de Montréal (1999-2003). 
Cartographie pour les 27 arrondissements. Direction de santé publique, Montréal, $158 \mathrm{p}$.

56. Environmental Systems Research Institute inc. (2010) ArcGIS Desktop 10.0. ESRI inc., Redlands.

57. Hirst W M, Mountain L J, Maher M J (2004) Sources of error in road safety scheme evaluation: A quantified comparison of current methods. Accid Anal Prev 36: 705-15.

58. Elvik R (1998) Are road safety evaluation studies published in peer reviewed journals more valid than similar studies not published in peer reviewed journals? Accid Anal Prev 30: 101-18.

59. SAS (2010) SAS version 9.2. SAS Institute Inc., Cary.

60. Hilbe J (2011) Negative binomial regression. Cambridge University Press, Cambridge, $541 \mathrm{p}$.

61. Miranda-Moreno L F, Morency P, El-Geneidy A M (2011) The link between built environment, pedestrian activity and pedestrianvehicle collision occurrence at signalized intersections. Accid Anal Prev 43: 1624-34.

62. Bressoux P (2008) Modélisation statistique appliquée aux sciences sociales. De Boeck, Bruxelles, $464 \mathrm{p}$.

63. Hosmer D, Lemeshow S (2000) Applied logistic regression. J. Wiley, New York, 392 p.
64. Tremblay M (2012) Évaluation du niveau d'activité et d'aménagement des intersections routières en lien avec les blessés piétons dans les quartiers centraux de Montréal. Rapport de projet de maîtrise, Université du Québec à Montréal, 113 p.

65. Linteau P A (1992) Histoire de Montréal depuis la Confédération. Boréal, Montréal, $613 \mathrm{p}$.

66. Demers M (2008) Pour une ville qui marche, aménagement urbain et santé. Les Éditions Écosociété, Montréal, $287 \mathrm{p}$.

67. Dumbaugh E, Rae R (2009) Safe urban form: Revisiting the relationship between community design and traffic safety. $J \mathrm{Am}$ Plann Assoc 75: 309-29.

68. Ewing R, Dumbaugh E (2009) The Built Environment and Traffic Safety. J Plan Lit 23: 347-67.

69. Organisation mondiale de la Santé (2013) Résumé: Rapport de situation sur la sécurité routière dans le monde. OMS, Genève, $12 \mathrm{p}$.

70. Arrondissement Plateau Mont-Royal (2009) Plan de déplacement urbain: Se réapproprier le quartier, Montréal, $92 \mathrm{p}$.

71. Arrondissement Plateau Mont-Royal (2009) Premier plan de déplacement urbain du Plateau Mont-Royal: Plan d'action 2009-2010, Montréal, 77 p. 